\title{
Two implications of common models of microbial growth
}

\author{
Simon Brown ${ }^{1}$
}

(Received 30 July 2007; revised 5 December 2007)

\begin{abstract}
Analysis of a generalised growth equation shows that both the maximum growth rate of a microbial culture and the duration of the lag phase are related to each other and to the maximum growth. Similar relationships apply to growth expressions, such as the logistic and Gompertz models, that are special cases of the generalised model. Moreover, the same relationships are observed qualitatively in measurements of the growth of Salmonella species. These results may allow the characterisation of microbial growth with fewer parameters than is usually the case and imply the likelihood of a fundamental physiological interdependence between maximum growth rate, the duration of the lag time and the maximum growth.
\end{abstract}

See http://anziamj.austms.org.au/ojs/index.php/ANZIAMJ/article/view/340 for this article, (c) Austral. Mathematical Soc. 2007. Published December 23, 2007. ISSN 1446-8735 


\section{Contents}

1 Introduction

C231

2 Parameter relationships

C232

3 Application to bacterial growth

C235

4 Conclusion

C239

References

C239

\section{Introduction}

Microbial population growth in liquid culture is triphasic. An initial period of little growth (the lag phase) is followed by a phase of rapid growth (the exponential phase) which eventually ceases and the population enters stationary phase (Figure 1). The lag phase is usually said to reflect the adaptation of the cells to the new environment and its duration $\lambda$ is often used to reflect this process. The maximum growth rate $\mu_{m}$ is measured from the exponential phase and the maximum growth $k$ is observed in the stationary phase. The growth in the size of the population $x(t)$ as a function of time $t$ has been modelled using

$$
x^{\prime}=\frac{\beta}{k^{n}} x^{1-n p}\left(k^{n}-x^{n}\right)^{p+1},
$$

where $\beta$ is related to $\mu_{m}, \lambda$ and $k$, and $n>0$ and $-1<p \leq 1 / n$ are constants [11] modulating the detailed shape of the growth curve. For convenience, the solution to (1) is

$$
x(t)=\frac{k}{\left[1+\left(\left\{x_{0}^{n} /\left(k^{n}-x_{0}^{n}\right)\right\}^{p}+\beta n p t\right)^{-1 / p}\right]^{1 / n}},
$$


where the initial inoculum is $x_{0}=x(0)$ and $t$ is the time following inoculation of the culture. Section 2 assumes that $p>0$ (or that $p \rightarrow 0$ ) in order to avoid the possible poles in (2). This form of the generalised growth equation (1) is related to other models $[6,12]$, and it incorporates several well known models as special cases. These include the logistic equation [13], when $n=1$ and $p=0$

$$
x^{\prime}=\frac{\beta}{k} x(k-x),
$$

the Malthusian model [5], if $k \rightarrow \infty$ and $p=0$

$$
x^{\prime}=\beta x,
$$

and the Gompertz model [4], for which $\beta n \rightarrow \beta^{\prime}, n \rightarrow 0$ and $p \rightarrow 0$

$$
x^{\prime}=\beta^{\prime} x \ln (k / x) .
$$

The Gompertz model is often used to obtain estimates of $k, \mu_{m}$ and $\lambda$ from microbial growth data [15], but (1) has previously been used to model the growth of rats [11] and cultured mammalian cells [7].

Of the parameters of interest to experimentalists (Figure 1), only $k$ appears in (1) and (2). The other parameters commonly used in the characterisation of microbial growth, $\mu_{m}$ and $\lambda$, are derived from (1) and related to each other, $k$ and $x_{0}$. Here two implications are drawn from (1): $k \propto \mu_{m} \lambda$ and $\mu_{m} \propto 1 / \lambda$ (Section 2). Moreover, these relationships are seen (Section 3) in at least some of the available microbial growth data, such as those in ComBase $[1,2]$.

\section{Parameter relationships}

Expressions relating the maximum growth rate $\mu_{m}$ and the duration of the lag phase $\lambda$ as functions of $\beta, k, x_{0}, n$ and $p$ are determined from (1). From 


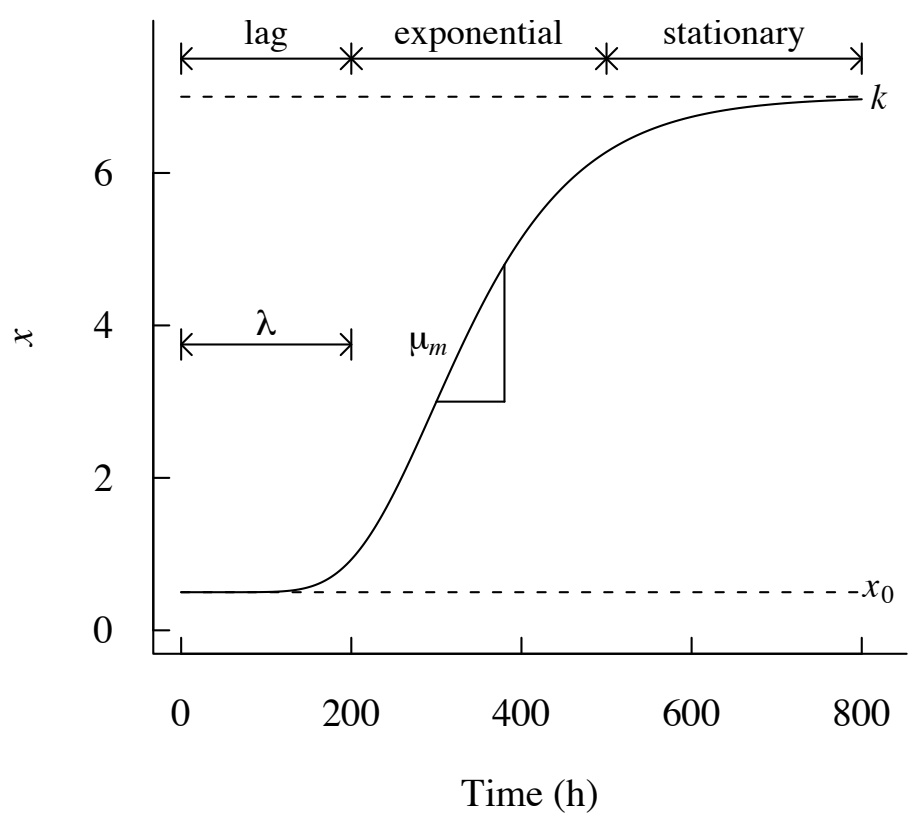

FiguRE 1: The triphasic growth curve and the parameters often employed to describe growth. The three phases are indicated approximately (top), the maximum growth $k$ and initial inoculum $x_{0}$ are indicated by horizontal dashed lines and the lag time $\lambda$ and maximum growth rate $\mu_{m}$ are also shown. 
these, an expression relating $\mu_{m}, \lambda$ and $k$ is obtained, which shows that $k \propto \mu_{m} \lambda$ and $\mu_{m} \propto 1 / \lambda$.

The maximum growth rate is the maximum value of $x^{\prime}$, which occurs when $x^{\prime \prime}=0$ and $x_{0}<x<k$,

$$
\mu_{m}=\left\{[n(p+1)]^{p+1}\left[\frac{(1-n p)^{(1-n p)}}{(1+n)^{(1+n)}}\right]^{1 / n}\right\} \beta k,
$$

and the term in braces depends only on $n$ and $p$, so (3) is $\mu_{m}=\alpha(n, p) \beta k$. For the logistic model $\alpha(1, p \rightarrow 0)=1 / 4$ and for the Gompertz model $\alpha(n \rightarrow 0, p \rightarrow 0)=1 / e$, as reported by Winsor [14].

The lag time $\lambda$ has been defined in at least two different ways: as the time at which the tangent to the exponential phase of the curve intersects $x=x_{0}$ [15]; or as the time at which $x^{\prime \prime}$ is a maximum [3]. The former yields an awkward expression (except in specific cases), but the latter definition yields

$$
\lambda=\frac{1}{n p \beta}\left[\left(\frac{\rho}{k^{n}-\rho}\right)^{p}-\left(\frac{x_{0}^{n}}{k^{n}-x_{0}^{n}}\right)^{p}\right],
$$

where $\rho$ is the $x^{n}$ value corresponding to the time at which the maximum of $x^{\prime \prime}$ occurs and is the smaller root of the quadratic in $x^{n}$

$$
(1+n)(1+2 n) x^{2 n}+(1+n)((3 p-1) n-2) k^{n} x^{n}+(n p-1)(2 n p-1) k^{2 n}=0 .
$$

Since $\rho$ depends only on $k, x_{0}, n$ and $p,(4)$ is

$$
\lambda=\frac{1}{\beta} g\left(k, x_{0}, n, p\right)
$$

which means, for example, that

$$
g\left(k, x_{0}, 1, p \rightarrow 0\right)=\ln \frac{a\left(k-x_{0}\right)}{x_{0}},
$$


where $a=(3-\sqrt{3}) /(3+\sqrt{3})$, for the logistic model, so $g\left(k, x_{0}, 1, p \rightarrow 0\right)$ increases with $k$.

Eliminating $\beta$ from (3) and (6), yields

$$
\mu_{m} \lambda=k \alpha(n, p) g\left(k, x_{0}, n, p\right)=k f\left(k, x_{0}, n, p\right),
$$

which defines the relationship implicit in (1) between the four experimentally relevant parameters. For the logistic model, an explicit form of (8) is

$$
\mu_{m} \lambda=\frac{k}{4} \ln \frac{a\left(k-x_{0}\right)}{x_{0}},
$$

obtained by substituting (7) and $\alpha(1, p \rightarrow 0)$ into (8). In this case, $\mu_{m} \lambda$ increases with $k$, as is also the case for the Gompertz model, although the expression is more complex.

\section{Application to bacterial growth}

The data shown in Figure 2 represent the growth of Salmonella species and illustrate some of the variation of $k(6<k<12), \mu_{m}\left(0 \mathrm{~h}^{-1}<\mu_{m} \leq\right.$ $\left.1 \mathrm{~h}^{-1}\right)$ and $\lambda(2 \mathrm{~h} \leq \lambda \leq 500 \mathrm{~h})$. The parameters estimated from more than 100 growth curves are plotted in Figure 3 according to (8). Most of the data conform to (8), and in those instances that do not (the yellow squares in Figure 3) at least one parameter was not reliably estimated. Most of these cases involved unreliable estimates of $k$, which occurred because the stationary phase was poorly defined by the data, as exemplified by the two growth curves in Figure 2 with the highest $\mu_{m}$ (the open squares and yellow diamonds).

The maximum growth rate $\mu_{m}$ should be proportional to $k$, consistent with (3), because both parameters have been shown to depend on the energy utilised per unit growth [10]. Specifically, $\mu_{m}$ is related to the ratio of the rate 


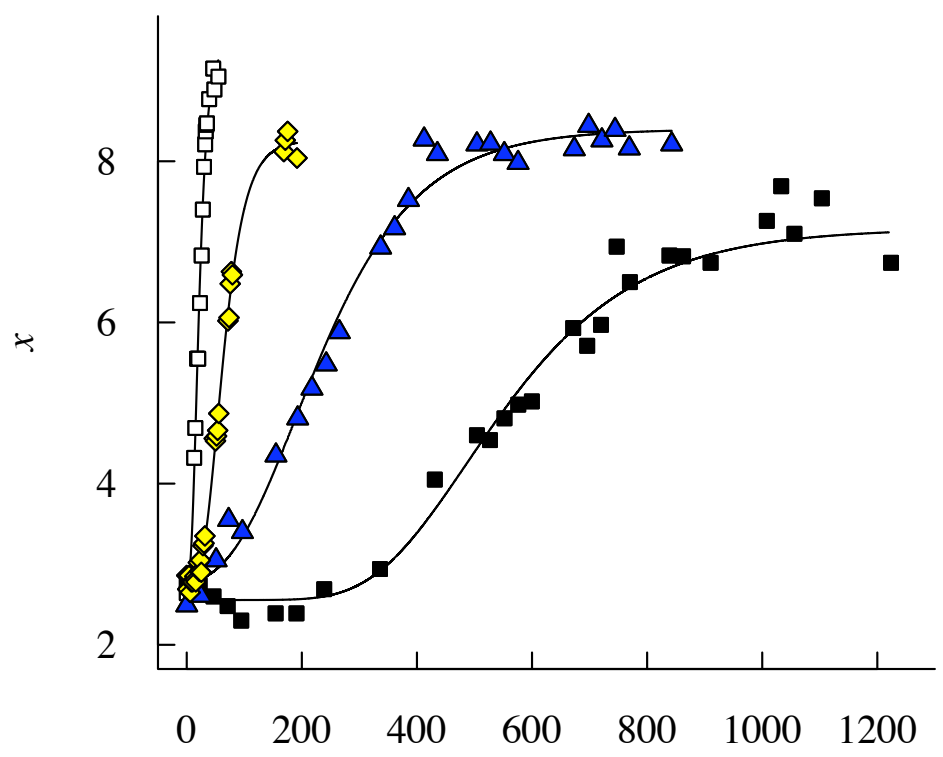

Time (h)

Figure 2: Examples of Salmonella sp. growth data selected from ComBase [1] (entry B092) to illustrate the variation in the parameters $(\lambda$, $\mu_{m}$ and $k$, that are analysed further in Figures 3 and 4 ) and in the quality of the data and the curves fitted to them [9]. The colour of the data points and the symbols used are intended only to differentiate between growth curves. 


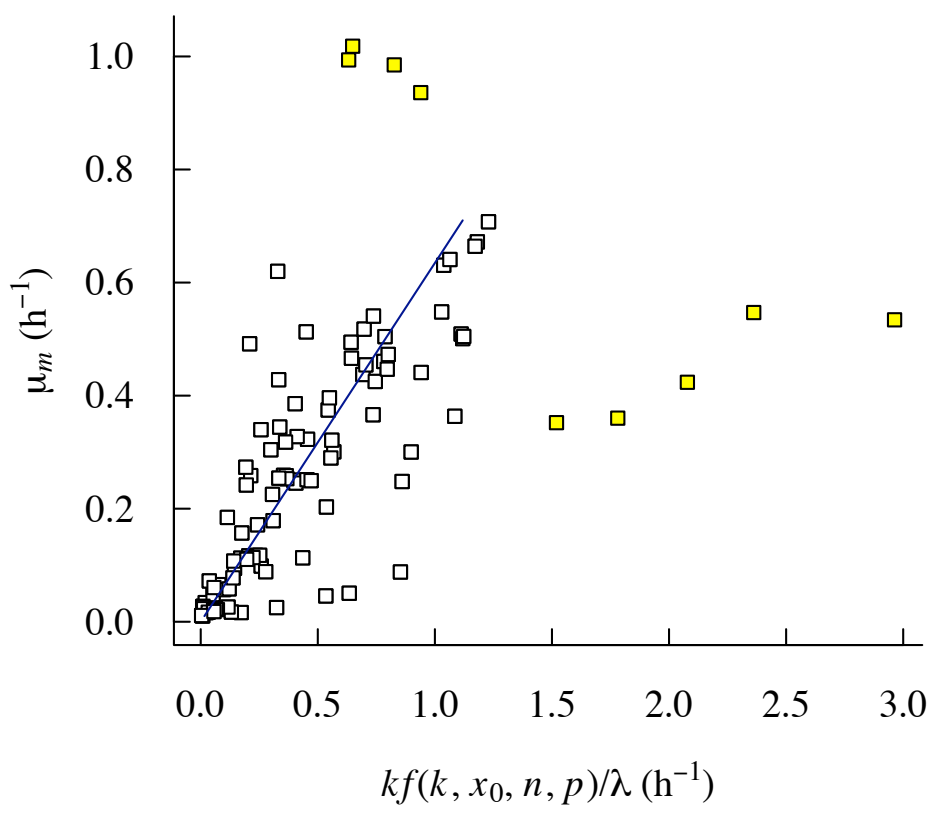

FigURE 3: Summary of the relationship between $k f\left(k, x_{0}, n, p\right)$ and $\mu_{m}$ (8) for 108 growth curves [1] (entry B092). The yellow squares represent data excluded from the analysis because at least one of the parameter estimates (generally $k$ ) was not reliable. 


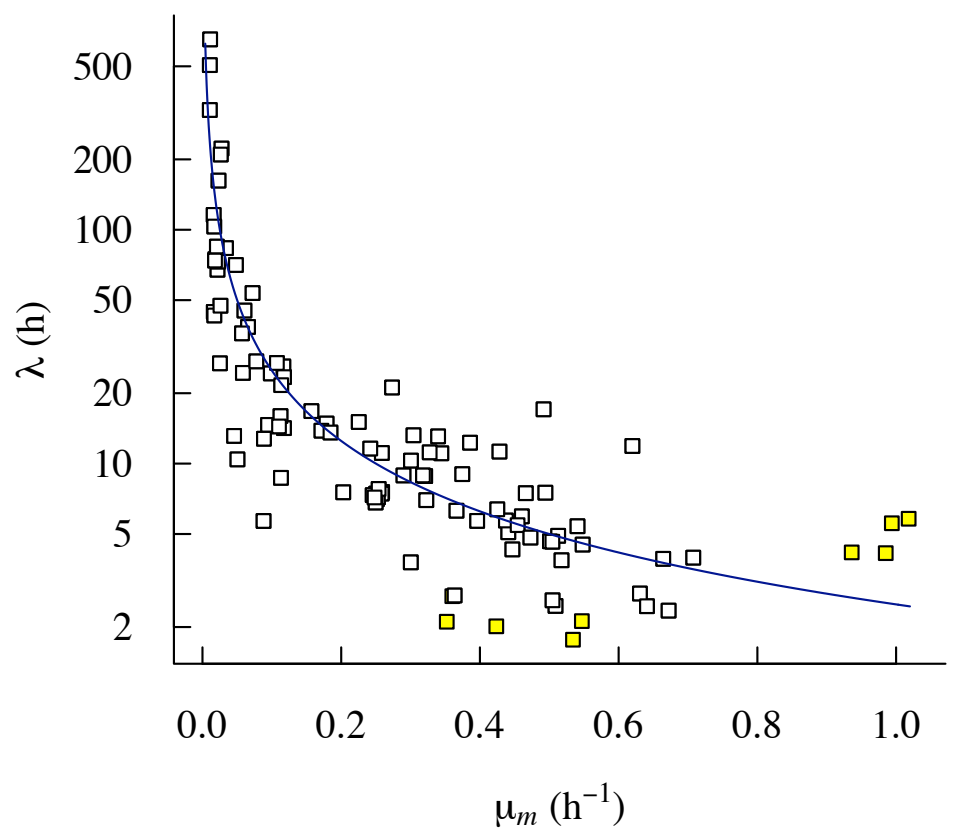

Figure 4: Summary of the relationship between $\lambda$ and $\mu_{m}$ for 108 growth curves [1] (entry B092). The yellow squares represent those data excluded from the analysis in Figure 3 because at least one of the parameter estimates was not reliable.

of energy utilisation and the energy utilised per unit growth [10]. The maximum growth $k$ depends on the composition of the medium and the efficiency with which the cells utilise the available energy to generate biomass [10], although it is also influenced by other factors, such as the sensing of population density [8].

Equation (8) indicates that $\mu_{m} \propto 1 / \lambda$, consistent with the data shown in Figure 4 . The duration of the lag phase $\lambda$ is usually related to the adaptation of the cells to a new growth environment. Several factors could contribute to a prolonged lag phase, for example the cells used to inoculate the culture could 
be in a poor physiological state, the population could be dominated by slowgrowing cells or the environment might be unsuitable. In such circumstance the cells might well be expected to exhibit a low $\mu_{m}$.

\section{Conclusion}

The growth of bacteria (Figure 2) has been modelled using (1), from which it has been shown that $\mu_{m} \propto k((3)$ and Figure 3$)$ and that $\mu_{m} \propto 1 / \lambda((8)$ and Figure 4). This implies that the faster a culture can grow $\mu_{m}$, the shorter the time required for the population to adapt to a new culture medium $\lambda$ and the greater the extent of growth $k$. As (1) includes several common growth models as special cases, these relationships are also observed in commonly used models of microbial growth [15]. The experimental confirmation of these relationships would indicate that the processes of adaptation and growth are functionally interdependent and that microbial growth can be characterised with fewer parameters.

\section{References}

[1] Anonymous, Combase. http://www. combase.cc. C232, C236, C237, C238

[2] Baranyi, J. and Tamplin, M. L., ComBase: a common database on microbial responses to food environments, J. Food Protect., 67, 2004, 1967-1971. http://apt.allenpress . com/aptonline/?request= get-abstract\&issn=0362-028X\&volume $=067 \&$ issue $=09 \& p a g e=1967$. $\mathrm{C} 232$ 
[3] Buchanan, R. L. and Cygnarowicz, M. L., A mathematical approach toward defining and calculating the duration of the lag phase, Food Microbiol., 7, 1990, 237-240. doi:10.1016/0740-0020(90)90029-H. C234

[4] Gompertz, B., On the nature of the function expressive of the law of human mortality, and on a new mode of determining the value of life contingencies, Phil. Trans R. Soc. Lond., 115, 1825, 513-585. doi:10.1098/rstl.1825.0026. C232

[5] Malthus, T., An Essay on the Principle of Population, as it Affects the Future Improvement of Society with Remarks on the Speculations of Mr. Godwin, M. Condorcet, and Other Writers, J. Johnson, 1798. $\mathrm{C} 232$

[6] Marusic, M. and Bajzer, Z., Generalized two-parameter equation of growth, J. Math. Anal. Appl., 179, 1993, 446-462.

doi:10.1006/jmaa.1993.1361. C232

[7] Marusic, M., Bajzer, Z., Vuk-Pavolic, S. and Freyer, J. P., Tumor growth in vivo and as multicellular spheroids compared by mathematical models, Bull. Math. Biol., 56, 1994, 617-631. doi:10.1007/BF02460714. C232

[8] Redfield, R. J., Is quorum sensing a side effect of diffusion sensing?, Trends Microbiol., 10, 2002, 365-370. doi:10.1016/S0966-842X(02)02400-9. C238

[9] R Core Development Team, R: a package for statistical computing, R Foundation for Statistical Computing, 2006. http://www.r-project.org. C236

[10] Russell, J. B. and Cook, G. M., Energetics of bacterial growth: balance of anabolic and catabolic reactions, Microbiol. Rev., 59, 1995, 48-62. http://www. pubmedcentral.nih.gov/articlerender.fcgi? artid $=239354$. C235, C238 
[11] Turner, M. E., Jr, Bradley, E. L., Jr, Kirk, K. A. and Pruitt, K. M., A theory of growth, Math. Biosci., 29, 1976, 367-373. doi:10.1016/0025-5564(76)90112-7. C231, C232

[12] Savageau, M. A., Growth equations - a general equation and a survey os special cases, Math. Biosci., 48, 1980, 267-278. doi:10.1016/0025-5564(80)90061-9. C232

[13] Verhulst, P.-F., Notice sur la loi que la population suit dans son accroissement, Corresp. Math. Physique, 10, 1838, 113-121. http://www.google.com.au/books?id=NTgDAAAAQAAJ\&printsec= frontcover\&dq=editions : OX2PwfU_YZepVwHW. C232

[14] Winsor, C. P., The Gompertz curve as a growth curve. Proc. Natl Acad. Sci. USA, 18, 1932, 1-8. http://www.pubmedcentral.nih. gov/articlerender.fcgi?artid=1076153. C234

[15] Zweitering, M. H., Jongenburger, I., Rombouts, F. M. and van't Riet, K., Modeling of the bacterial growth curve, Appl. Environ. Microbiol., 56, 1990, 1875-1881.

http://intl-aem.asm.org/cgi/content/abstract/56/6/1875. C232, C234, C239 


\section{Author address}

1. Simon Brown, School of Human Life Sciences, University of Tasmania, Launceston, Australia.

mailto:Simon.Brown@utas. edu . au 